\title{
A Case of Fanconi's Syndrome in a Patient with HIV
}

\author{
Mariam Kabir MD, PhD, Christopher Miller MD, Rakesh Gulati, MD, MRCP, \\ William R. Short MD, MPH
}

\section{INTRODUCTION}

Tenofovir disoproxil fumarate (TDF) is a nucleotide analogue reverse transcriptase inhibitor (NtRTI), which blocks reverse transcriptase, an enzyme found in HIV. Since its approval for use in HIV by the FDA in 2001, it has contributed to effective treatment in numerous patients. The most common side effects include nausea, vomiting, diarrhea, asthenia, abdominal pain and hepatotoxicity. A less common side effect is nephrotoxicity leading to Fanconi's syndrome. Here is an interesting case of Fanconi's Syndrome caused by Tenofovir.

\section{CASE}

A 50-year-old Caucasian female with a past medical history of HIV and Hepatitis C presented to the Emergency Department with hypokalemia and acute renal failure. She had been diagnosed with HIV in 2003 and was being managed on co-formulated Truvada (Emtracitabine/Tenofovir) and Efavirenz since 2008. She was previously on Lamivudine/Zidovudine and Efavirenz, which were discontinued due to side effects. Over the past month, the patient was noted to have hypokalemia and worsening serum creatinine ( $\mathrm{s} C \mathrm{r}$ ), which was being treated with potassium supplements and avoidance of NSAIDs. On presentation she denied any diuretic use, nausea, vomiting, diarrhea, weakness, fatigue, paralysis, palpitations, syncope, lightheadedness or chest pain.

The patient's HIV was under good control (last CD4 count of 1400 cells $/ \mathrm{mm} 3$ and viral load undetectable at $<20$ copies $/ \mathrm{ml}$ ), and her viral load for hepatitis $C$ was negligible (HCV RNA quantitative real time PCR $<43$ IU/ $\mathrm{ml}$ ). She did not have any history of seizure disorder, refractory migraine or use of drugs such as zonisamide or carbonic anhydrase inhibitors. Pertinent medications included Truvada 1 Tablet every 48 hours and Efavirenz $600 \mathrm{mg}$ at bedtime. She had no drug allergies. Social history was only positive for 1 pack per day of cigarette use for many years.

On physical exam the patient was afebrile and her vital signs were stable. She appeared to be in no apparent distress. She was alert and oriented to time, place and self. She did not have any scleral icterus or thrush in her throat. She had moist mucous membranes. Her pulmonary, cardiovascular, abdominal and neurological exams were normal. She did not have any costrovertebral angle tenderness. She also had no pedal edema.

Her laboratory data were as follows: sodium $135 \mathrm{mmol} / \mathrm{L}$ (normal 135-146mmol/L), potassium $1.9 \mathrm{mmol} / \mathrm{L}$ (normal 3.5-5mmol/L), chloride $97 \mathrm{mmol} / \mathrm{L}$ (89-109 $\mathrm{mmol} / \mathrm{L}$ ), bicarbonate $22 \mathrm{mmol} / \mathrm{L}$ (24-32 mmol/L), BUN $20 \mathrm{mg} / \mathrm{dL}$ (normal 7-26 mg/dL), creatinine1.7 mg/dL (0.7-1.4 mg/dL), anion gap $16 \mathrm{mmol} / \mathrm{L}(4-16 \mathrm{mmol} / \mathrm{L})$, magnesium $1.9 \mathrm{mEq} / \mathrm{L}(1.3-2.1 \mathrm{mEq} / \mathrm{L})$, phosphate 2 $\mathrm{mg} / \mathrm{dL}$ (2.4-4.5 mg/dL)(low). Urinalysis showed yellow urine with a pH of 7.0, 1+ Glucose, 1+ protein, urine potassium $13 \mathrm{mmol} / \mathrm{L}$, urine osmolality $211 \mathrm{mmol} / \mathrm{L}$, serum osmolality $293 \mathrm{mmol} / \mathrm{L}$. Serum creatinine and potassium in 2008 were $0.8 \mathrm{mg} / \mathrm{dL}$ and $3.6 \mathrm{mmol} / \mathrm{L}$ respectively, and $1 \mathrm{mg} / \mathrm{dL}$ and $3.5 \mathrm{mmol} / \mathrm{L}$ six months ago. Renal ultrasound showed mildly echogenic kidneys suggesting renal parenchymal disease.

\section{DIFFERENTIAL DIAGNOSIS AND HOSPITAL COURSE}

Given her glycosuria, proteinuria, hypophosphatemia in addition to hypokalemia and acute renal failure, a diagnosis of renal tubular acidosis type 2 and Fanconi's Syndrome was made. The etiology of this was thought to be her Tenofovir use (part of the Truvada combo); she had been receiving this for the past 5 years. Based on our consulting nephrologists recommendations, her Truvada was discontinued, her potassium was repleted with both intravenous and oral supplements, and her 
renal function improved with intravenous hydration. At the time of discharge her Creatinine was $1.1 \mathrm{mg} / \mathrm{dL}$ and her potassium was $2.8 \mathrm{mmol} / \mathrm{L}$. She was discharged with oral potassium supplementation and appropriate follow up with her infectious disease doctor.

\section{DISCUSSION}

Tenofovir is available in combination with Emtricitabine in a product with the brand name Truvada. It is also available in a single tablet regimen, Atripla, which contains Tenofovir, Emtricitabine and Efavirenz. Tenofovir is the only NtRTI approved for use in HIV. ${ }^{1}$ It is also approved for the treatment of chronic hepatitis B and as pre-exposure prophylaxis against HIV infection. ${ }^{1}$

Tenofovir has been deemed a suitable NtRTI secondary to less adverse effects on blood lipids and less mitochondiral toxicity. It was also found to have less renal toxicity in early randomized clinical trials. ${ }^{2}$ Although initially there were concerns given Tenofovir's molecular/ structural similarity to other nucleotide analogs Adefovir and Cidofovir, which cause proximal tubulopathy by decreasing mitochondrial DNA replication (inhibits mtDNA polymerase), it was found that mtDNA depletion was negligible with Tenofovir. ${ }^{3}$ However in other studies, Tenofovir has been shown to be nephrotoxic to proximal tubular cells. ${ }^{4}$ The discrepancy is thought to be secondary to the strict inclusion and exclusion criteria applied in the clinical trials. ${ }^{4}$

Nephrotoxicity from Tenofovir can result in proximal tubular dysfunction with normal renal function, or with acute or chronic renal failure. The renal failure can happen months to years after starting the drug. The major renal biopsy finding is proximal tubular injury, ranging from mild and localized to diffuse and severe. This is associated with varying degrees of chronic tubulointerstitial scarring (i.e., tubular atrophy and interstitial fibrosis). ${ }^{5}$

The proximal tubulopathy results in Fanconi's syndrome, either partial or complete. ${ }^{6}$ Complete Fanconi's syndrome consists of renal tubular acidosis, glycosuria with hypophosphatemia, aminoaciduria, hypouricemia and tubular proteinuria. Renal function decline may follow tubular dysfunction.7 B2-microglobinuira is prevalent. ${ }^{8}$ Patients may have osteomalacia secondary to phosphate wasting and calcitriol deficiency, since calcitriol is absorbed in the mitochondria of proximal tubules. ${ }^{7.9}$ Our patient in the above-mentioned case had most of these findings.

The incidence and prevalence of Tenofovir toxicity is varied. A study conducted in 2006 by a Spanish medical group found that $22 \%$ of tenofovir-treated patients had tubular dysfunction as opposed to $12 \%$ of naive HIV patients and $6 \%$ of HAART-treated patients. A retrospective study of HIV-infected patients on Tenofovir identified $1 \%$ whose $\mathrm{s} C \mathrm{r}$ increased. ${ }^{10}$

Thus, in order to manage patients with HIV on Tenofovir, one must be able to figure out who is at risk of nephrotoxicity. Postmarketing clinical data analysis showed that advanced age, low body weight, higher serum creatinine levels before starting Tenofovir treatment, comorbidities (diabetes, hypertension, HCV coinfection), concomitant nephrotoxic medications, advanced HIV infection (low CD4 counts, AIDS), and male sex were risk factors for Tenofovir-induced GFR reduction. 2,10,11

\section{FUTURE DIRECTIONS}

Tenofovir alafenamide (TAF) is a pro-drug of Tenofovir and is currently in Phase III clinical trials. ${ }^{12}$ Compared to Tenofovir, TAF has increased HIV-1 activity, increased intracellular Tenofovir diphosphate levels by 7 fold, decreased circulating plasma Tenofovir levels by $90 \%$, which results in lower levels of Tenofovir in kidney and bone tissue. This decrease will hopefully cause less renal toxicity as well as decreased bone mineral toxicity. In a recent phase 2 randomized, placebocontrolled trial, treatment naive HIV-1 infected individuals were randomized 2:1 to receive Elvitegravir/ Cobicistat/Emtricitabine/TAF or Elvitegravir/Cobicistat/ Emtricitabine/Tenofovir. In the 24 week analysis, those receiving the compound with TAF had high levels of virologic suppression as well as a significantly smaller increase in serum creatinine and smaller decreases in bone mineral density of the hip and spine. 
